\title{
Quantitative law describing market dynamics before and after interest-rate change
}

\author{
Alexander M. Petersen, ${ }^{1}$ Fengzhong Wang, ${ }^{1}$ Shlomo Havlin, ${ }^{1,2}$ and H. Eugene Stanley ${ }^{1}$ \\ ${ }^{1}$ Center for Polymer Studies and Department of Physics, Boston University, Boston, Massachusetts 02215, USA \\ ${ }^{2}$ Minerva Center and Department of Physics, Bar-Ilan University, Ramat-Gan 52900, Israel
}

(Received 7 December 2009; revised manuscript received 3 May 2010; published 28 June 2010)

\begin{abstract}
We study the behavior of U.S. markets both before and after U.S. Federal Open Market Commission meetings and show that the announcement of a U.S. Federal Reserve rate change causes a financial shock, where the dynamics after the announcement is described by an analog of the Omori earthquake law. We quantify the rate $n(t)$ of aftershocks following an interest-rate change at time $T$ and find power-law decay which scales as $n(t-T) \sim(t-T)^{-\Omega}$, with $\Omega$ positive. Surprisingly, we find that the same law describes the rate $n^{\prime}(|t-T|)$ of "preshocks" before the interest-rate change at time $T$. This study quantitatively relates the size of the market response to the news which caused the shock and uncovers the presence of quantifiable preshocks. We demonstrate that the news associated with interest-rate change is responsible for causing both the anticipation before the announcement and the surprise after the announcement. We estimate the magnitude of financial news using the relative difference between the U.S. Treasury Bill and the Federal Funds effective rate. Our results are consistent with the "sign effect," in which "bad news" has a larger impact than "good news." Furthermore, we observe significant volatility aftershocks, confirming a "market under-reaction" that lasts at least one trading day.
\end{abstract}

DOI: 10.1103/PhysRevE.81.066121

PACS number(s): 89.65.Gh, 91.30.Px, 89.75.Da, 64.60.av

\section{INTRODUCTION}

Interest-rate changes by the Federal Reserve provide a significant perturbation to financial markets, which we analyze from the perspective of statistical physics [1-6]. The Federal Reserve Board (Fed), in charge of monetary policy as the central bank of the United States, is one of the most influential financial institutions in the world. During Federal Open Market Commission (FOMC) meetings, the Fed determines whether or not to change key interest rates. These interest rates serve as a benchmark and a barometer for both American and international economies. The publicly released statements from the scheduled FOMC meetings provide grounds for widespread speculation in financial markets, often with significant consequences.

In this paper, we show that markets respond sharply to FOMC news in a complex way reminiscent of physical earthquakes described by the Omori law $[7,8]$. For financial markets, the Omori law was first observed in market crashes by Lillo and Mantegna [9], followed by a further study of Weber et al. [10], which found the same behavior in medium-sized aftershocks. However, the market crash is only an extreme example of information flow in financial markets. This paper extends the Omori law observed in large financial crises to the more common Federal Reserve announcements and suggests that large market news dissipates via power-law relaxation (Omori law) of the volatility. In addition to the standard Omori dynamics following the announcement, we also find unique Omori dynamics before the announcement.

The market dynamics following the release of FOMC news are consistent with previous studies of price discovery in foreign exchange markets following marcroeconomic news releases $[11,12]$. Furthermore, we hypothesize that the uncertainty in Fed actions, coupled with the preannounced schedule of FOMC meetings, can increase speculation among market traders, which can lead to the observed market under-reaction [13]. Market under-reaction, meaning that markets take a finite time to readjust prices following news, is not consistent with the efficient market hypothesis; several theories have been proposed to account for these phenomena [14].

We analyze all 66 scheduled FOMC meetings in the 8 year period of 2000-2008 using daily data from [15]. Also, for the 2 year period of 2001-2002, we analyze the intraday behavior for 19 FOMC meetings using Trades and Quote (TAQ) data on the 1 min time scale.

The paper is organized as follows. In Sec. II we describe the FOMC meetings and the Fed interest rate relevant to our analysis. In Sec. III A we analyze the response of the S\&P 100 , the top 100 stocks (ranked by 12 month sales according to a 2002 BusinessWeek report) belonging to the $2002 \mathrm{~S} \& \mathrm{P}$ 500 index, over the 2000-2008 period using daily data. Using the relative spread between the 6 month Treasury Bill and the Federal Funds effective rates, we relate the speculation prior to the FOMC meetings to the daily market volatility, measured here as the logarithmic difference between the intraday high and low prices for a given stock on the day of the announcement. In Sec. III B we study high-frequency intraday TAQ data on the $1 \mathrm{~min}$ scale for the S\&P 100 and find an Omori law with positive exponent immediately following the announcement of Fed rate changes. Further, we relate the intraday market response (quantified by both the Omori exponent and Omori amplitude) to the change in market expectations before and after the announcement.

\section{FOMC MEETINGS, FED INTEREST RATES, AND TREASURY BILLS}

There are many economic indicators that determine the health of the U.S. economy. In turn, the health of the U.S. economy sets a global standard due to the ubiquity of both 
the U.S. Dollar and the economic presence maintained through imports, exports, and the global market [16]. The U.S. Federal Reserve target rate, along with the effective "overnight" rate, sets the scale for interest rates in the United States and abroad. The target rate is determined at FOMC meetings, which are scheduled throughout the year, with detailed minutes publicly released from these meetings. The effective rate is a "weighted average of rates on brokered trades" between the Fed and large banks and financial institutions and is a market realization of the target rate [17]. In Fig. 1 we plot the federal interest rates over the 8 year period of 2000-2008.

Our analysis focuses on the FOMC meetings after January 2000. Historically, the methods for releasing the meeting details have varied. In the 1990s, there was a transition from a very secretive policy toward the current transparent policy [18]. Since the year 2000, the Fed has released statements detailing the views and goals of the FOMC. This increase in public information has led to an era of mass speculation in the markets, revolving mainly around key economic indicators such as the unemployment rate, the consumer price index, the money supply, etc. These economic indicators also influence the FOMC in their decision to either change or maintain key interest rates. Speculation has assumed many forms and new heights, evident in the implementation of new types of derivatives based on federal securities. For instance, options and futures are available at the Chicago Board of Trade, which are based on Federal Funds, Treasury Bills, and Euro-Dollar foreign exchange. These contracts can be used to estimate the implied probability of interest-rate changes, utilizing sophisticated methods focused on the price movement of expiring derivative contracts [19-24].

In the next section, we outline a simple method to measure speculation prior to a scheduled FOMC meeting using the 6 month Treasury Bill and the Federal Funds effective (overnight) rate. These data are readily available and are updated frequently at the website of the Federal Reserve [17]. Because each FOMC meeting is met with speculation (in the weeks before the meeting) and anticipation (in the hours before the announcement), we identify the decision to change or not to change key interest rates as a market perturbation. The market response results from the systematic stress associated with the speculation and anticipation, which are not always in line with the FOMC decision.

\section{EMPIRICAL RESULTS}

\section{A. Response to FOMC meetings on daily time scale}

In this section we analyze the daily activity before and after 66 scheduled FOMC meetings over the 8 year period of 2000-2008, where scheduled meetings are publicly announced at least a year in advance [17]. We do not consider unscheduled meetings resulting in rate change, which contain an intrinsic element of surprise and are historically infrequent (only four unexpected target rate changes over the same period). Of primary importance is the FOMC committee's decision to change or not change the target rate $R(t)$ by some percent $\Delta R(t)$, where the absolute relative change $|\Delta R(t) / R(t-1)|$ has typically filled the range between 0.0 and
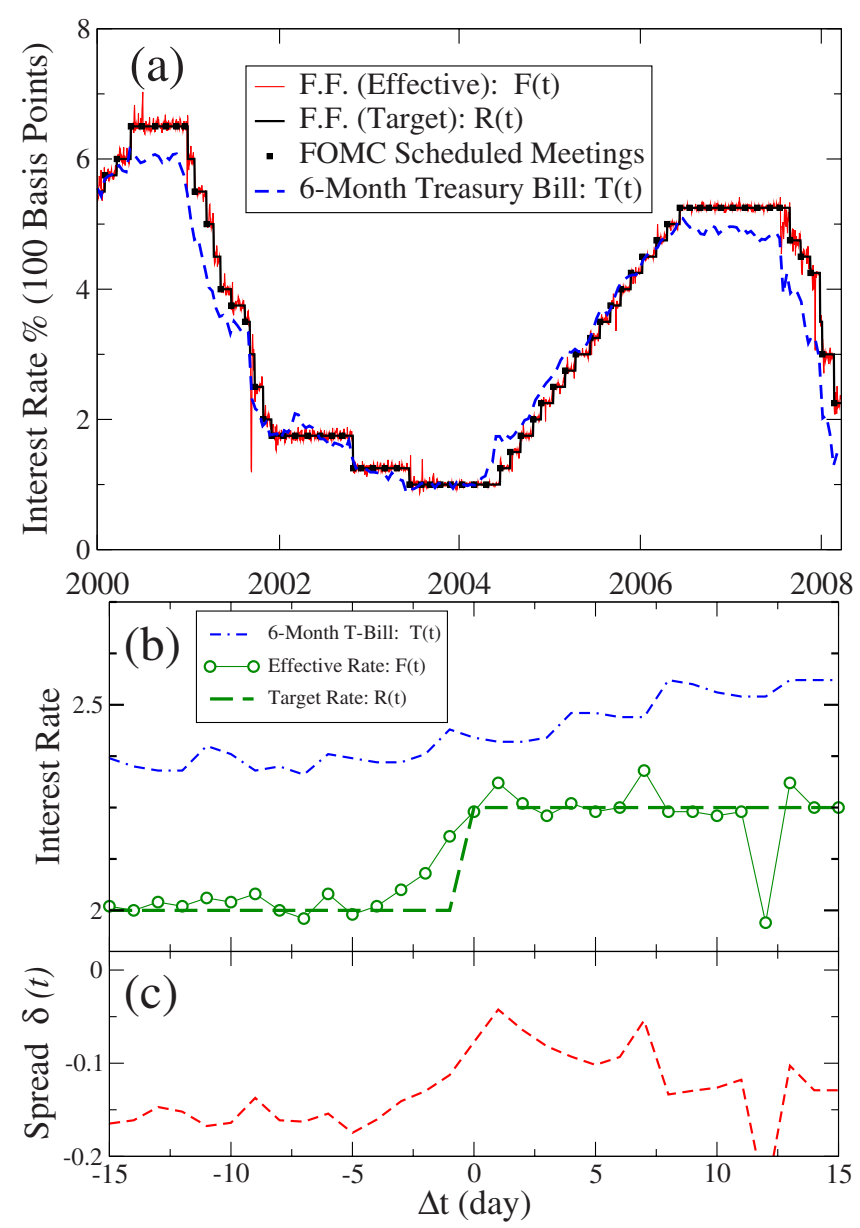

FIG. 1. (Color online) An illustration of the close relation between the Treasury Bill and the Federal Funds rate. (a) Time series of the Federal Reserve Target rate $R(t)$ and the Federal Reserve Effective rate $F(t)$ for Federal Funds dating from January 2000 to April 2008. The 6 month Treasury Bill $T(t)$ closely follows the effective rate, with speculation about future changes causing deviations in the relative values. United States Treasury Bills carry little risk and are considered to be one of the most secure investments. (b) A typical illustration of the Federal Funds effective rate and the Treasury Bill, where both gravitate around the Federal Funds target rate. The change in the relative spread $\delta(t)$, defined in Eq. (1), between the Treasury Bill and the Federal Funds effective rate, indicates changes in market speculation. (c) The relative spread $\delta(t), 15$ days before and 15 days after the scheduled FOMC meeting on December 14, 2004, which corresponds to $\Delta t=0$. Note that the average value of the relative spread increases after the announcement, indicating a shift in market consensus and speculation.

0.25 . This section serves as an initial motivation for the intraday analysis and will also serve as a guide in developing a metric that captures market speculation. In this section we use the intraday high-low price range to quantify the magnitude of price fluctuations. In particular, we analyze the companies belonging to the S\&P 100 and also the subset of 18 banking and finance companies referred to here as the "bank" sector.

In Fig. 1(a) we plot $T(t)$, the time series for the 6 month Treasury Bill, along with $F(t)$, the Federal Funds effective rate, and $R(t)$, the Federal Funds target rate, over the 8 year 
period beginning in January 2000. The relative difference between the 6 month Treasury Bill and the Federal Funds effective rate is an indicator of the future expectations of the Federal Funds target rate [18]. Note that the 6 month Treasury Bill has anticipatory behavior with respect to the Federal Funds target (and hence effective) rates. Other more sophisticated models utilize futures on Federal Funds and Euro-Dollar exchange, but these markets are rather new and represent the highly complex nature of contemporary markets and hedging programs [20-24]. Hence, we use a simple and intuitive method for estimating market speculation and anticipation by analyzing the relative difference between the 6 month Treasury Bill and the Federal Funds effective rate.

Figure 1(b) exhibits the typical interplay between the 6 month Treasury Bill and the Federal Funds effective rate before and after a FOMC meeting. The change in the value of the effective rate results from market speculation, starting approximately one trading week (five trading days) prior to the announcement. This change follows from the forwardlooking Treasury Bill, which in the example in Fig. 1(b), is priced above the Federal Funds rate even 15 trading days before the announcement.

In order to quantify speculation and anticipation in the market prior to each scheduled FOMC meeting, we analyze the time series $\delta(t)$ of the relative spread between $F(t)$ and $T(t)$,

$$
\delta(t) \equiv \ln \left(\frac{F(t)}{T(t)}\right) .
$$

As an example of this relation, in Fig. 1(c) we plot $\delta(t)$ for 15 days before and after a typical FOMC meeting resulting in a rate change. In order to study the speculation preceding the $i$ th scheduled FOMC meeting, we calculate the average relative spread over the $L_{1}=15$ day period. We weight the days in the $L_{1}$ day period leading up to the FOMC meeting day exponentially, such that the relative spread on the $(\Delta t)$ th day before the announcement has the weight $w(\Delta t)=e^{-\Delta t / \lambda}$. Without loss of generality, we choose the value of $\lambda$ $=10$ days corresponding to two trading weeks [25]. We define the speculation metric,

$$
\Theta_{i}=\overline{\delta(t)_{i}} \equiv \frac{\sum_{\Delta t} \delta\left(t_{i}-\Delta t\right) w(\Delta t)}{\sum_{\Delta t} w(\Delta t)},
$$

which is a weighted average of $\delta(t)$ before the announcement, where the sums are computed over the range $\Delta t$ $\in\left[1, L_{1}\right]$. The metric $\Theta_{i}$ for the $i$ th FOMC meeting can be positive or negative, depending on the market's forwardlooking expectations.

In order to quantify the market response to the speculation $\Theta_{i}$, we analyze the market volatility around each FOMC meeting. For a particular stock around the $i$ th scheduled FOMC meeting, we take the daily high price $p_{\mathrm{hi}}\left(t_{i}+\Delta t\right)$ and the daily low price $p_{\text {low }}\left(t_{i}+\Delta t\right)$, for $\Delta t \in[-20,20]$, where $\Delta t=0$ corresponds to $t_{i}$, the day of the meeting. We then compute the high-low range for each trading day,

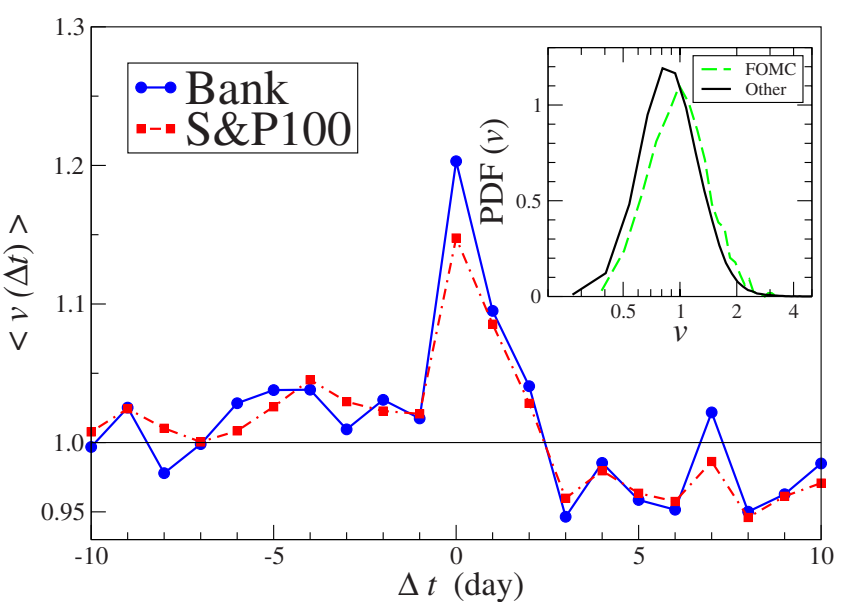

FIG. 2. (Color online) Average daily volatility trend defined in Eq. (4) exhibits increased market volatility on the day of FOMC meetings, corresponding to $\Delta t=0$. "Bank" refers to the portfolio of 18 stocks that belong to the S\&P 100. There is a $15-20 \%$ increase in volatility on days corresponding to FOMC meetings. Standard deviation $\sigma(v(\Delta t)) \approx 0.4$ can be assigned to each data point in the time series and is calculated by randomizing the daily volatility time series of each company. (Inset) Probability density function (pdf) of normalized volatility $v \equiv r(t) /\langle r\rangle$, where the quantity $r(t)$ $\equiv \ln \left[p_{\text {hi }}(t) / p_{\text {low }}(t)\right]$ is the range of the price time series of a given stock for a particular day. We plot the pdf of volatility values for the S\&P 100 on the set of days with FOMC meetings and for the set of all "other" days. The distributions are approximately logarithmic normal, with a shift toward higher average volatility on FOMC days. The average values for the two data sets are $\langle v\rangle_{F O M C}=1.12$ and $\langle v\rangle_{\text {other }}=1.00$.

$$
r\left(t_{i}+\Delta t\right) \equiv \ln \left(\frac{p_{\mathrm{hi}}\left(t_{i}+\Delta t\right)}{p_{\text {low }}\left(t_{i}+\Delta t\right)}\right) .
$$

For each stock and each meeting, we scale the range by $\langle r\rangle$, the average range over the 41 day time sequence centered around the meeting day, resulting in the normalized volatility $v\left(t_{i}+\Delta t\right) \equiv r\left(t_{i}+\Delta t\right) /\langle r\rangle$. Similarly, we use $\Phi\left(t_{i}+\Delta t\right)$, the time series for the volume traded over the same period, to compute a weight for each stock corresponding to the normalized volume on the day of the FOMC meeting. We calculate this weight as $\phi_{i} \equiv \Phi\left(t_{i}\right) /\langle\Phi\rangle_{i}$, where $\langle\Phi\rangle_{i}$ is the average daily volume over the 41 day time sequence centered around the $i$ th meeting day. We use a volume weight in order to emphasize the price impact resulting from relatively high trading volume, since there are significant cross correlations between volume and price changes [26]. Finally, we compute the weighted average volatility time series over all stocks and all meetings,

$$
\langle v(\Delta t)\rangle \equiv \frac{\sum v\left(t_{i}+\Delta t\right) \phi_{i}}{\sum \phi_{i}} .
$$

In Fig. 2 we plot the trend of average daily volatility defined in Eq. (4) for 10 days before and after the scheduled announcements.

We observe a peak in $\langle v(\Delta t)\rangle$ on FOMC meeting days, corresponding to $\Delta t=0$, with a more pronounced peak in the 
bank sector (Fig. 2). Stocks in the bank sector are strongly impacted by changes in Fed rates, which immediately influence both their holding and lending rates. On average there is a $15-20 \%$ increase in volatility on days corresponding to FOMC meetings.

In order to quantify the impact of a single FOMC announcement on day $t_{i}$, we define the average market volatility,

$$
V_{i}=\left\langle v\left(t_{i}\right)\right\rangle \equiv \frac{\sum^{(i)} v\left(t_{i}\right) \phi_{i}}{\sum \phi_{i}} .
$$

Here, $\langle\cdots\rangle_{i}$ and $\Sigma^{(i)}$ refer to the average and sum over records corresponding only to the day $t_{i}$. Again, $\phi_{i}$ $\equiv \Phi\left(t_{i}\right) /\langle\Phi\rangle$ is a normalized weight, where now $\langle\Phi\rangle$ is the average daily volume over the entire 8 year period, since we compare many meetings across a large time span.

In Fig. 3 we plot the average volatility $V_{i}$ of the S\&P 100 [Fig. 3(a)] and the subset of 18 banking stocks [Fig. 3(b)] versus $\Theta_{i}$. For negative values of $\Theta_{i}$, for which $T(t)>F(t)$ corresponding to an expected rate increase, we observe a less volatile market response. Conversely, for larger positive values of $\Theta_{i}$, for which $T(t)<F(t)$ corresponding to a rate cut, there tends to be larger market fluctuations. Hence, the market responds differently to falling and rising rates, where the direction in rate change often reflects the overall health of the economy as viewed by the FOMC. Typically, the FOMC implements rate increases to fight inflation, whereas rate decreases often follow bad economic news or economic emergency. Hence, our findings are consistent with the empirical sign effect, in which "bad" news has a greater impact in markets than does "good" news [12]. Furthermore, there is also a tendency for large average volatility even when $\Theta_{i}$ is small, possibly stemming from the extreme surprise characteristic of some FOMC decisions. In these cases, more sophisticated methods are needed to improve the predictions of market movement.

\section{B. Intraday response to FOMC decision via an Omori law}

In the previous section we studied the market response on the daily scale. Now we ask the question "what is the intraday response to FOMC news?" Here, we analyze the TAQ data over the 2 year period from January 1, 2001 to December 31, 2002. The reported times for the FOMC announcement are listed in Table I [27]. Inspired by the nonstationary nature of financial time series, methods have been developed within the framework of nonequilibrium statistical mechanics to describe phenomena ranging from volatility clustering [28-30] to financial correlation matrices [31-33].

We use the Omori law, originally proposed in 1894 to describe the relaxation of aftershocks following earthquakes, to describe the response of the market to FOMC announcements. Defined in Ref. [9], the Omori law quantifies the rate $n(t)$ of large volatility events following a singular perturbation at time $T$. The shock may be exogenous (resulting from external news stimuli) or endogenous (resulting from internal
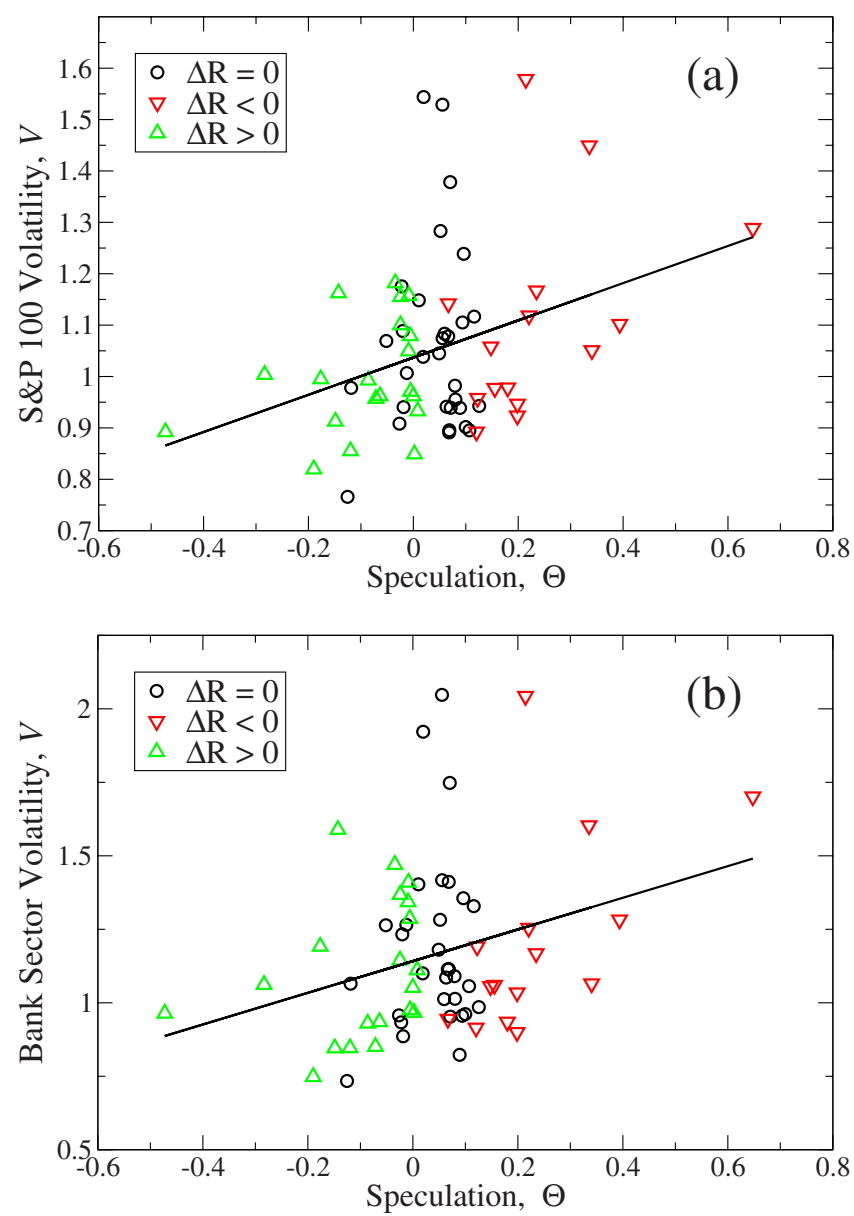

FIG. 3. (Color online) Demonstration of the relation between speculation of interest-rate change and market volatility in the $S \& P$ 100 and for the subset of banking stocks. We relate $\Theta_{i}$, the speculation in the market before a FOMC meeting defined in Eq. (2), to the market volatility $V_{i}$ defined in Eq. (5). A large absolute value of $\Theta_{i}$ reflects the high probability that an interest-rate change will happen. Interestingly, there are many instances where $\Theta_{i} \approx 0$, followed by large volatility. These values correspond to FOMC decisions to maintain interest-rate levels $(\Delta R=0)$ and suggest a fundamental difference in the dynamics following decisions to change versus decisions not change the Federal Funds target rate. Also, there is an underlying symmetry in $\Delta R$ since in the case of either a rate increase or a rate decrease, the FOMC also has the option of no increase. Hence, $\Delta R=0$ can occur as either good or bad news, whereas typically decisions of $\Delta R>0$ reflect situations with positive market sentiment whereas decisions of $\Delta R<0$ reflect situations with negative market sentiment. Hence, the asymmetry in market volatility is consistent with the sign effect [12]. Although the correlation between $\Theta_{i}$ and $V_{i}$ is dominated by residual error, it is nevertheless supporting that the regression captures the crossover at $(\theta, V)=(0,1)$. Including all data points, the regression correlation coefficient is $r^{2}=0.34$, and the slope of the regression is $m$ $=0.36 \pm 0.13$ for (a), and $r^{2}=0.30$ and $m=0.54 \pm 0.22$ for (b). Restricting data points corresponding only to interest-rate changes (red and green triangles): $r^{2}=0.48$ and $m=0.37 \pm 0.12$ for (a), and $r^{2}$ $=0.40$ and $m=0.53 \pm 0.21$ for (b) (this second regression is not shown and is indistinguishable from the regression including all data points). All linear regressions pass the ANOVA (Analysis of variance) $F$ test, rejecting the null hypothesis that $m=0$ at the $\alpha$ $=0.05$ significance level. 
TABLE I. Reported times of market perturbations in the form of FOMC news. Dates of 19 FOMC meetings in the 2 year period between January 2001 and December 2002, where the Federal Funds Target rate $R_{\text {new }}$ was implemented by the rate change $\Delta R$ at $T$ minutes after the opening bell at 9:30 a.m. ET. The absolute relative change $\left|\Delta R / R_{\text {old }}\right| \equiv|\Delta R(t) / R(t-1)|$ has typically filled the range between 0.0 and 0.25 . Note that date** refers to unscheduled meetings, in which the announcement time did not correspond to 2:15 p.m. ET $(T=285 \mathrm{~min})$ [27].

\begin{tabular}{lllcr}
\hline \hline FOMC date & $\begin{array}{c}R_{\text {new }} \\
(\%)\end{array}$ & $\Delta R$ & $\frac{\Delta R}{R_{\text {old }}}$ & \multicolumn{1}{c}{$T$} \\
\hline $01 / 03 / 01^{* *}$ & 6 & -0.5 & -0.077 & 210 \\
$01 / 31 / 01$ & 5.5 & -0.5 & -0.083 & 285 \\
$03 / 20 / 01$ & 5 & -0.5 & -0.091 & 285 \\
$04 / 18 / 01^{* *}$ & 4.5 & -0.5 & -0.100 & 90 \\
$05 / 15 / 01$ & 4 & -0.5 & -0.111 & 285 \\
$06 / 27 / 01$ & 3.75 & -0.25 & -0.063 & 285 \\
$08 / 21 / 01$ & 3.5 & -0.25 & -0.067 & 285 \\
$09 / 17 / 01^{* *}$ & 3 & -0.5 & -0.143 & 0 \\
$10 / 02 / 01$ & 2.5 & -0.5 & -0.167 & 285 \\
$11 / 06 / 01$ & 2 & -0.5 & -0.200 & 285 \\
$12 / 11 / 01$ & 1.75 & -0.25 & -0.125 & 285 \\
$01 / 30 / 02$ & 1.75 & 0 & 0.00 & 285 \\
$03 / 19 / 02$ & 1.75 & 0 & 0.00 & 285 \\
$05 / 07 / 02$ & 1.75 & 0 & 0.00 & 285 \\
$06 / 26 / 02$ & 1.75 & 0 & 0.00 & 285 \\
$08 / 13 / 02$ & 1.75 & 0 & 0.00 & 285 \\
$09 / 24 / 02$ & 1.75 & 0 & 0.00 & 285 \\
$11 / 06 / 02$ & 1.25 & -0.5 & -0.286 & 285 \\
$12 / 10 / 02$ & 1.25 & 0 & 0.00 & 285 \\
\hline \hline
\end{tabular}

correlations, e.g., "herding effect") [34-38]. This rate is defined as

$$
n(|t-T|) \sim|t-T|^{-\Omega},
$$

where $\Omega$ is the Omori power-law exponent.

Here, we study the rate of events greater than a volatility threshold $q$, using the high-frequency intraday price time series $p(t)$. The intraday volatility (absolute returns) is expressed as $v(t) \equiv|\ln [p(t) / p(t-\delta t)]|$, where we use $\delta t$ $=1 \mathrm{~min}$. To compare stocks, we scale each raw time series in terms of the standard deviation over the entire period analyzed, and then remove the average intraday trading pattern as described in Ref. [10]. This establishes a common volatility threshold $q$, in units of standard deviation, for all stocks analyzed.

In the analysis that follows, we focus on $N(|t-T|)$, the cumulative number of events above threshold $q$,

$$
N(|t-T|)=\int_{T}^{t} n\left(\left|t^{\prime}-T\right|\right) d t^{\prime} \equiv \beta(|t-T|)^{1-\Omega},
$$

which is less noisy compared to $n(|t-T|)$. Using $N(|t-T|)$, we examine the intraday market dynamics for $100 \mathrm{~S} \& \mathrm{P}$ stocks before $(t<T)$ and after $(t>T)$ the $i$ th FOMC an- nouncement at $T_{i}$, which typically occurs at 2:15 p.m. eastern time (ET) (285 min after the opening bell) for scheduled meetings.

In Figs. 4(a) and 4(b) we plot the average volatility response $N(t)$ of the $S \equiv 100$ stocks analyzed, where

$$
N(t) \equiv \frac{1}{S} \sum_{j=1}^{S} N^{j}(t) .
$$

This average is obtained by combining the individual Omori responses, $N^{j}(t)$, of the $S$ stocks. Such averaging does not cancel the Omori law, but allows for better statistical regression. This is especially useful for an Omori law corresponding to large volatility threshold $q$, where a single stock might not have a sufficient number of events. In Fig. 4(c) we plot the trade pattern $N^{j}(t)$ of Merrill Lynch (MER) on Tuesday 08/21/01, and also in Fig. 5 for the following 3 days, demonstrating that the Omori relaxation can persists for several days.

The abrupt change in the curvature of $N(t)$ illustrates the volatility clustering which begins around the time of the announcement $T$, corresponding to the vertical line at $t$ $=285$ min in Figs. 4(a)-4(c). For comparison, we find that the average $\langle N(t)\rangle$ time series calculated from all days without FOMC meetings is approximately linear with time throughout the entire day, indicating that the sudden increase in excess volatility before and after announcement times $T$ results from the FOMC news. Volatility clustering in financial data sampled at the $1 \mathrm{~min}$ scale persists for several months, with a significant crossover in the observed powerlaw autocorrelations occurring around $600 \mathrm{~min}(\approx 1.5$ days) [39-41].

In order to compare the dynamics before and after the announcement, we first separate the intraday time series $N(t)$ into two time series $N_{b}(t \mid t<T)$ and $N_{a}(t \mid t>T)$. Then, to treat the dynamics symmetrically around the $i$ th intraday announcement time $T_{i}[38,42]$, we define the displaced time $\tau$ $=\left|t-T_{i}\right| \geq 1$ as the temporal distance from the minute $T_{i}$ [43]. As an illustration, we plot $N(\tau)$ in Fig. 6 for the four corresponding $N(t)$ curves exhibited in Fig. 4(a). We then employ a linear fit to both $N_{b, i}(\tau)=N_{i}\left(T_{i}\right)-N_{i}\left(\left|t-T_{i}\right|\right)$ and $N_{a, i}(\tau)$ $=N_{i}\left(t-T_{i}\right)-N_{i}\left(T_{i}\right)$ on a log-log scale to determine the Omori power-law exponents $\Omega_{b}$ before the news and $\Omega_{a}$ after the news. In analogy, we define the amplitude $\beta$ before as $\beta_{b}$ and after as $\beta_{a}$, as defined in Eq. (7).

Typically $\Omega_{a}>0$, which reflects the pronounced increase in the rate of events above the volatility threshold $q$ after the time of the announcement. We also observe $\Omega<0$, which corresponds to a time series in which the preshocks or aftershocks farther away from the announcement (for large $\tau$ ) are dominant over the volatility cascade around time $\tau \approx 0$. For comparison, $n(\tau)$ is constant for stochastic processes with no memory, corresponding to $\Omega \equiv 0$. Hence, for an empirical value $\Omega \approx 0$, the rate $n(\tau)$ is indistinguishable from an exponential decay for $\tau<\bar{t}$, where $\bar{t}$ is the characteristic exponential time scale. However, for larger values of $\Omega$, the exponential and power-law response curves are distinguishable, especially if several orders of magnitude in $\tau$ is available. 
(a) S\&P100 (Volatility)

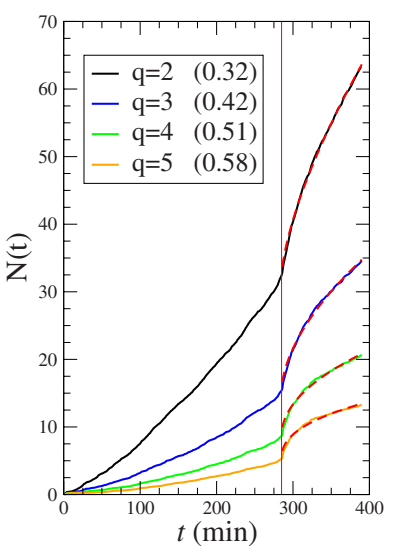

(c) MER (Volatility)

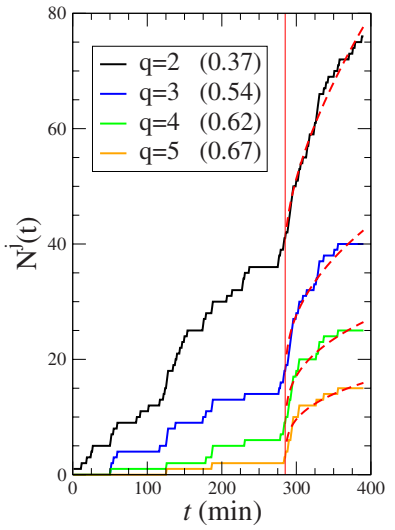

(d)

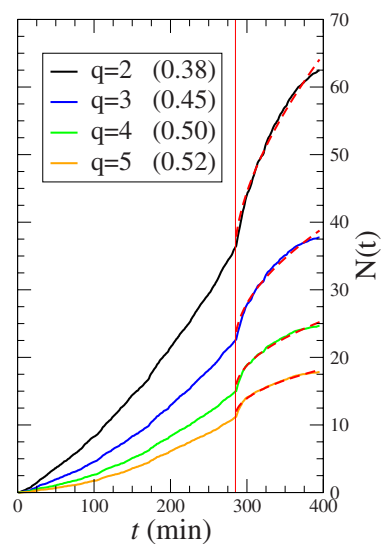

d) Bank Sector (Volatility)

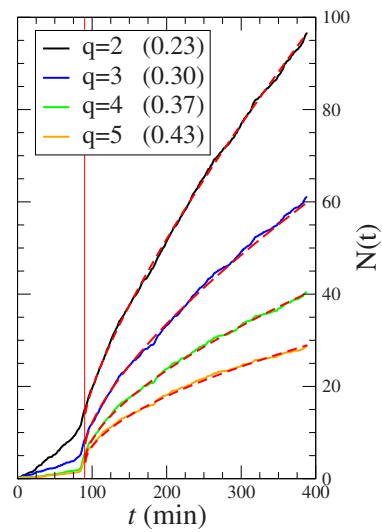

(b) S\&P100 (Volume)

FIG. 4. (Color online) The cumulative volatility time series $N(t)$ demonstrates Omori-law response dynamics; here in response to FOMC announcements occurring at the time $T$ indicated by a vertical solid red line. The abrupt change in the curvature of $N(t)$ around time $t \approx T$ illustrates the increased volatility caused by the announcement. The significant aftershocks which occur until the end of the trading day are consistent with market under-reaction $[11,12]$. Market under-reaction and other market inefficiencies can result from increased levels of uncertainty among traders following market news [13]. Each time series $N(t)$ is calculated for a given volatility threshold $q$, where larger $q$ values correspond to $N(t)$ curves with smaller amplitude (smaller rate of large volatility events). (a)-(c) illustrate the dynamics around a scheduled announcement made at $T=285 \min (2: 15$ p.m. ET). For the S\&P 100, we calculate $N(t)$ on $05 / 15 / 01$ for (a) 1 min volatility and (b) 1 min total volume using Eq. (8). (c) We calculate $N^{j}(t)$ for MER on 08/21/01. (d) The Omori law also occurs for unscheduled FOMC announcements, as illustrated for the bank sector $N(t)$ on $04 / 18 / 01$, when the surprise rate change was announced at $T=90 \min$ (11:00 a.m. ET), resulting in raised levels of volatility throughout the entire trading day. For (a)-(d), the dashed red lines are power-law fits beginning immediately after the announcement, with the corresponding exponents $\Omega_{a}(q)$ appearing in parentheses within the legends.

For all meetings analyzed, we find that $\Omega \equiv \Omega(q)$ increases with $q$, meaning that the relatively large aftershocks decay more quickly than the relatively small aftershocks. Hence, the largest volatility values cluster around the an- (a) Bank Sector (Volatility)

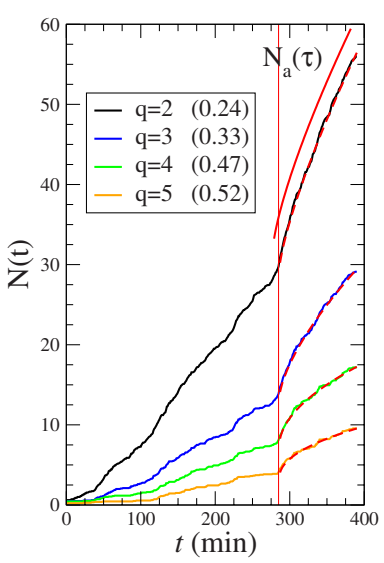

(b) MER (Volatility)

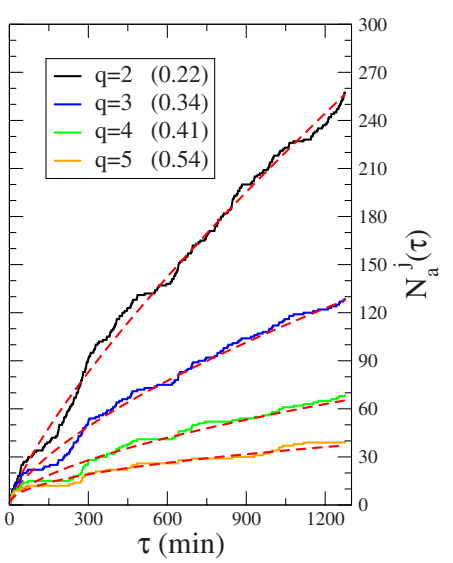

FIG. 5. (Color online) The Omori-law relaxation can extend for several days. We compare the Omori exponents $\Omega_{a}(q)$ (indicated in legends) calculated for (a) the time series $N_{a}(\tau)$ of the bank sector and (b) the time series $N_{a}^{j}(\tau)$ of Merrill Lynch for 3 days (1275 min) after the announcement on Tuesday $08 / 21 / 01$ at $2: 15$ p.m. $(\tau$ $=0 \mathrm{~min}$ corresponding to $T=285 \mathrm{~min}$ ). For the remaining 3 days of the trading week, the Omori-law relaxation corresponding to an individual stock (MER) is quantitatively similar to the Omori-law relaxation of the bank sector over the final $105 \mathrm{~min}$ of the initial trading day. We do not use the bank sector $N_{a}(\tau)$ over the same 1275 min time period for comparison because "opening effects" occurring during the first $60 \mathrm{~min}$ of each trading day make powerlaw regression of conjoined $N_{a}(\tau)$ problematic.

nouncement time $T$. For comparison, $\Omega(q)$ values are calculated in [9] using $q=4,5,6,7$ and in [10] using $q=3,4$ for large financial crashes. For our data set, the cumulative probability $P(v>q)$ that a given volatility value is greater than volatility threshold $q$ is $P(v>3)=0.18$ and $P(v>5)=0.087$. Furthermore, we reject the null hypothesis that $q>5$ volatilities are distributed evenly across all days, finding that $5 \%$ of
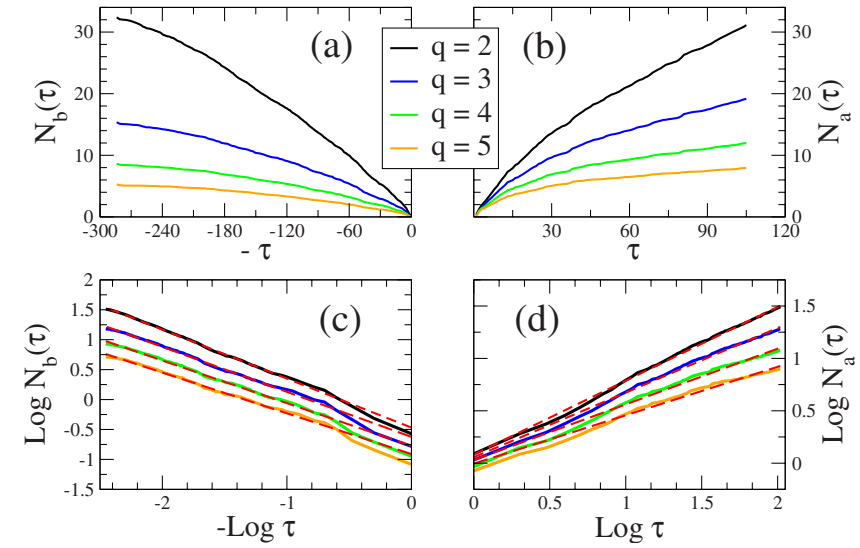

FIG. 6. (Color online) An illustration of the method used to calculate (a) $N_{b, i}(\tau)=N_{i}\left(T_{i}\right)-N_{i}\left(\left|t-T_{i}\right|\right)$ and (b) $N_{a, i}(\tau)=N_{i}\left(t-T_{i}\right)$ $-N_{i}\left(T_{i}\right)$ for each intraday time series $N_{i}(t)$. The displaced time $\tau$ $=\left|t-T_{i}\right|$ is defined symmetrically around the time of the announcement $T_{i}$. We plot the same data as in Fig. 4(a), corresponding to the announcement on $05 / 15 / 01$ which occurred at $T=285 \mathrm{~min}$. (c) and (d) show that $N_{b, i}(\tau)$ and $N_{a, i}(\tau)$ are approximately linear on logarithmic scale. 
the volatility values greater than $q=5$ are found on FOMC meeting days, whereas only $4 \%$ are expected under the null hypothesis that large volatilities are distributed uniformly across all trading days. The $25 \%$ increase for $q=5$ indicates that FOMC meetings days are more volatile than other days at the $\alpha \approx 0$ significance level. We also observe that the amplitudes of the Omori law generally obey the inequality $\beta_{b}$ $<\beta_{a}$, resulting from the large response immediately following the news.

Although we focus mainly on price volatility $v(t)$ in this paper, we also observe Omori dynamics in the highfrequency volume time series $\omega(t)$, defined as the cumulative number of shares traded in minute $t$. In Figs. 7(a)-7(d) for the S\&P 100 and Figs. 7(e)-7(h) for the bank sector, we compare the average of Omori exponents $\Omega_{b}$ and $\Omega_{a}$ for both volatility and volume dynamics, and for volatility threshold value $q=3$. We compute the average Omori exponents using two averaging methods: the "individual" method and the "portfolio" method.

To analyze the time series $N_{a, i}$ after the announcement $i$, we first average the exponents $\Omega_{a}^{j}$ obtained for each individual stock $j$, yielding $\left\langle\Omega_{a}\right\rangle$. This individual method provides an error bar corresponding to the sample standard deviation $\sigma\left(\Omega_{a}\right)$. The second portfolio method determines a single $\Omega_{a}$ from $N(t)$ in Eq. (8). Comparing the open-box (individual method) and closed-box (portfolio method) symbols in Fig. 7, we observe that both methods yield approximately the same average value of $\Omega_{a}$. Note that for the subset $i=\{1,4,8\}$ of the unscheduled FOMC meetings, $\Omega_{a}$ is smaller than usual, capturing the intense activity following surprise announcements. Hence, unexpected FOMC announcements can produce an inverse Omori law exhibiting convex relaxation $\left(\Omega_{a}<0\right)$ over a short horizon if the news contains a large amount of inherent surprise. The eight meeting corresponds to the opening of the markets after September 11, 2001.

For the time series $N_{b, i}$ before the announcement $i$, individual stocks often do not have sufficient activity to provide accurate power-law fits. Hence, to estimate the sample standard deviation $\sigma\left(\Omega_{b}\right)$, we produce partial combinations, $\langle N(\tau)\rangle_{b, i} \equiv \frac{1}{M} \sum_{j=1}^{M} N_{b, i}^{j}(\tau)$ using $M \equiv 5$. We then compute a standard deviation $\sigma\left(\Omega_{b}\right)$ from the $\Omega_{b}$ values calculated from $\langle N(\tau)\rangle_{b, i}$. The $\sigma\left(\Omega_{b}\right)$ values correspond to the error bars for $\left\langle\Omega_{b}\right\rangle$ in Fig. 7.

We also compute a single $\Omega_{b}$ value from the portfolio average $N_{b, i}(\tau)$, which corresponds to the limit $M=S$. The values of $\Omega_{b}$ using the two methods are consistent. Interestingly, the values of $\Omega_{b}$ calculated from volume data are all close to zero. However, using the student $T$ test we reject the null hypothesis that each average value $\left\langle\Omega_{b}\right\rangle$ is equal to zero at the $\alpha=0.01$ significance level for 15 out of 17 dates.

Figure 7 shows the range of $\Omega$ values for each of the 19 FOMC meetings we analyze. There are eight panels comparing the $\Omega$ values (i) between the dynamics before and after $T$, (ii) between the volatility and volume dynamics, and (iii) between set of all stocks comprising the S\&P 100 and the set of stocks comprising the banking sector. We hypothesize that the differences in the Omori $\Omega$ values, before and after the announcement, are related to the anticipation and perceived surprise of the FOMC news. Furthermore, for the dynamics
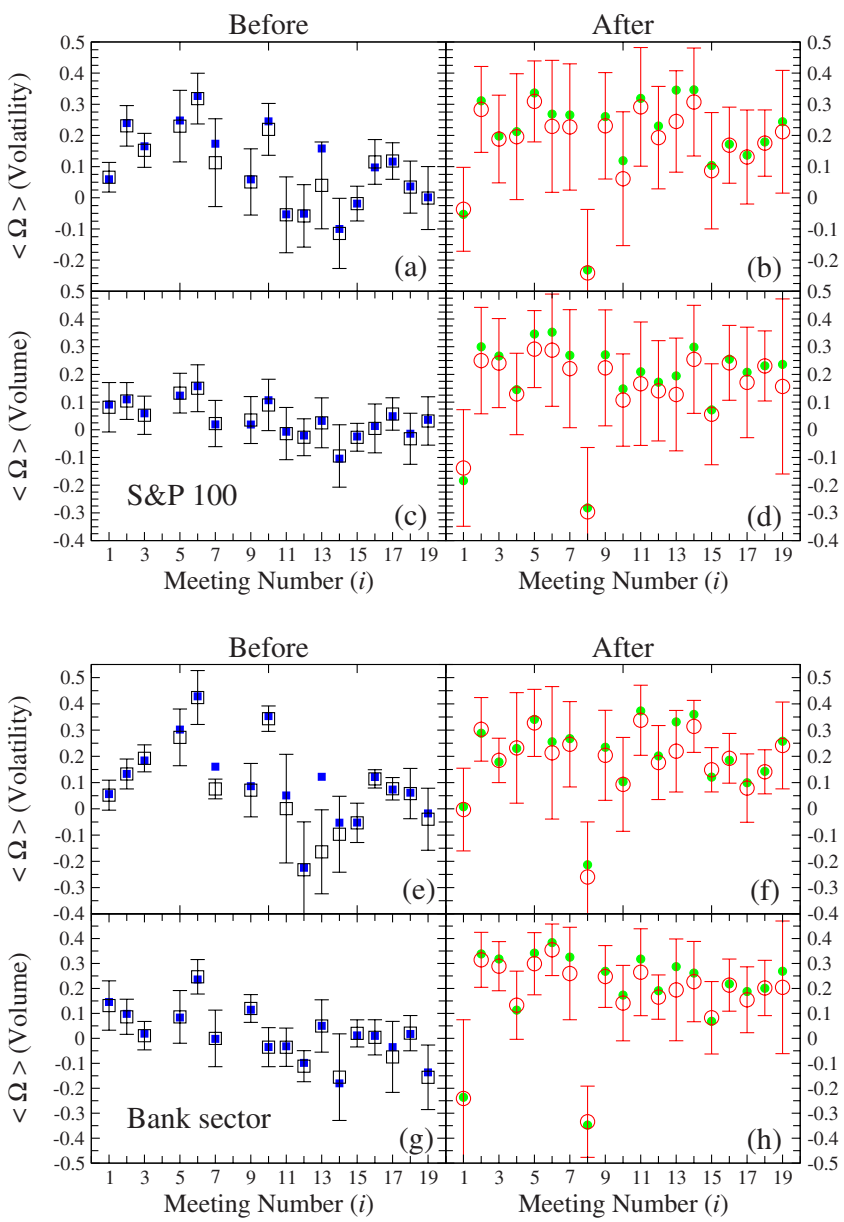

FIG. 7. (Color online) Comparison of Omori-law exponents for both volatility dynamics and volume dynamics on the day of 19 FOMC meetings during the 2 year period of January 2001December 2002. (a)-(d) correspond to the S\&P 100 and (e)-(h) correspond to the bank sector. The average value of $\Omega$ for the 16 scheduled FOMC meetings (excluding the three unannounced meetings $i=\{1,4,8\}$ ) are (a) $\bar{\Omega}_{b}=0.10 \pm 0.13$, (b) $\bar{\Omega}_{a}=0.24 \pm 0.08$, (c) $\bar{\Omega}_{b}=0.04 \pm 0.07$, (d) $\bar{\Omega}_{a}=0.24 \pm 0.07$, (e) $\bar{\Omega}_{b}=0.11 \pm 0.16$, (f) $\bar{\Omega}_{a}$ $=0.23 \pm 0.09$, (g) $\bar{\Omega}_{b}=0.01 \pm 0.10$, and (h) $\bar{\Omega}_{a}=0.26 \pm 0.08$. The similarity in exponents for $1 \mathrm{~min}$ volatility and $1 \mathrm{~min}$ cumulative volume suggests a universal underlying mechanism. Solid symbols ( $\square$ and $\bigcirc$ ) refer to $\Omega$ computed from $N(t)$. Open symbols ( $\square$ and $\bigcirc)$ refer to $\langle\Omega\rangle$ computed from $S$ individual Omori exponents $\Omega^{j}$, with $S_{\text {bank }}=18$. Note the relatively low values of $\Omega_{a}$ and $\left\langle\Omega_{a}\right\rangle$ for unscheduled FOMC announcements $i=1$ and 8 , which indicates that volatility rate following the announcement increased throughout the day.

after the news, we find anomalous negative $\Omega_{a}$ values for two surprise FOMC announcements $i=1$ and $i=8$. Also, we find that volume $\Omega$ values are more regular across all meeting events, suggesting that volume and price volatility contain distinct market information $[44,45]$.

In order to find potential variations in the response dynamics for different stock sectors, in Fig. 8(a) we compare the $\Omega_{a}$ values after the announcement for five approximately equal-sized sectors using volatility threshold $q=3$. We observe that the differences in the average values of the sectors 

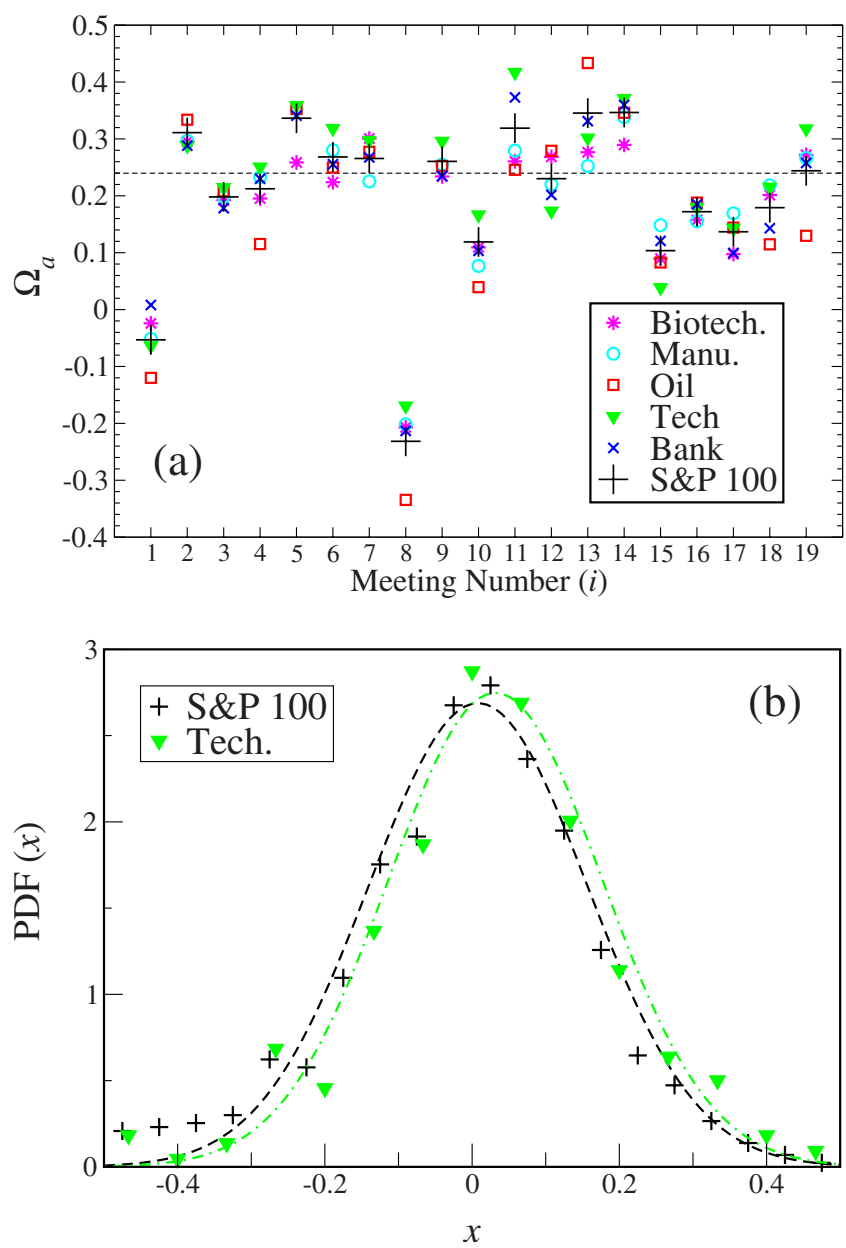

FIG. 8. (Color online) (a) A comparison of $\Omega_{a}$ for five sectors with volatility threshold $q=3$ suggests a broad universal market response to FOMC news. The technology sector tends to have the largest average $\Omega_{a}$, where large $\Omega$ values correspond to faster relaxation. The horizontal straight line represents the mean $\bar{\Omega}_{a}$ $=0.24 \pm 0.08$, averaged over all stocks in the S\&P 100 and all scheduled meetings (excluding the unscheduled meetings $i$ $=\{1,4,8\}$ ). (b) Probability density function $P(x)$ of the variable $x$ $\equiv x_{a, i}^{j}=\Omega_{a, i}^{j}-\left\langle\Omega_{a, i}\right\rangle$, which corresponds to individual $\Omega_{a, i}^{j}$ values centered around the average exponent $\left\langle\Omega_{a, i}\right\rangle$ of a given meeting $i$. We conclude from a $Z$ test at the $\alpha=0.0005$ significance level that technology sector Omori exponents are larger on average, $\langle x\rangle_{\text {Tech }}$ $>\langle x\rangle_{S P 100}$. Hence, since larger $\Omega$ values correspond to shorter relaxation time, we find that the technology sector stocks respond more quickly to FOMC news, possibly as a result of relatively intense trading activity among these stocks.

are fairly small, indicating a broad market response. We also observe that the technology sector, composed of hardware, software, and information technology (IT) companies, often has the largest average $\Omega_{a}$ value. Larger exponents, which correspond to shorter relaxation times, could result from the intense trading in the technology sector during the technology/IT bubble, which peaked in the year 2000. In follow-up analysis, we find in [46] that stocks with higher trading activity, quantified as the average number of transactions per minute, have larger $\Omega^{j}$ in response to market shocks and, thus, faster price discovery. In order to compare the variation in the individual values of $\Omega_{a}^{j}$, we plot the probability density function (pdf) of exponents for all stocks and meetings in Fig. 8(b) using the shifted variable $x^{j} \equiv \Omega_{a, i}^{j}$ $-\Omega_{a, i}$. We conclude from a $Z$ test at the $\alpha=0.0005$ significance level that technology sector Omori exponents are larger on average, $\left.\langle x\rangle_{\text {Tech }}\right\rangle\langle x\rangle_{S P 100}$.

Motivated by the metric $\Theta_{i}$ defined in Eq. (2), which quantifies speculation and anticipation in the market preceding FOMC meetings, we now develop a second metric to describe surprise through the change in market speculation following the announcement. This metric $\Delta_{i}$ compares the anticipation leading up to the announcement with the revised speculation following the FOMC decision. This can be quantified through the relative change in $\delta(t)$, which provides a rough measure of the market stress that is released in the financial shock. Qualitatively, $\Delta_{i}$ relates the average value of the spread before and after the $i$ th scheduled meeting. We define

$$
\begin{aligned}
\Delta_{i} & \equiv\left[\overline{\delta(t)}_{i, a}-\overline{\delta(t)}_{i, b}\right] S\left(\Delta R_{i}\right) \\
& \equiv\left(\frac{\sum \delta\left(t_{i}-\Delta t\right) w(\Delta t)}{\sum w(\Delta t)}-\frac{\sum \delta\left(t_{i}+\Delta t\right) w(\Delta t)}{\sum w(\Delta t)}\right) S\left(\Delta R_{i}\right),
\end{aligned}
$$

where the sum is computed over the range $\Delta t \in\left[1, L_{2}\right]$ trading days, with $L_{2}=15$ trading days and $\lambda_{2}=10$ trading days. The factor $S\left(\Delta R_{i}\right)=1$ when the Fed increases or maintains the target rate $R(t)$, while $S\left(\Delta R_{i}\right)=-1$ when the Fed decreases the target rate.

In Figs. 9(a)-9(d) we relate the amplitudes $\left\langle\beta_{b}\right\rangle$ and $\left\langle\beta_{a}\right\rangle$, and also the exponents $\left\langle\Omega_{b}\right\rangle$ and $\left\langle\Omega_{b}\right\rangle$, to the speculation metric $\Theta$ and the surprise metric $\Delta$. We observe that larger $\Theta$ and larger $\Delta$ are related to larger amplitude $\left\langle\beta_{b}\right\rangle$ quantifying the preshock dynamics. However, we do not find a statistically significant relation between $\Theta$ or $\Delta$ and the aftershock parameters, suggesting that the relaxation dynamics following FOMC news are less predictable. Nevertheless, the aftershock dynamics are consistently more pronounced, with $\left.\left\langle\beta_{a}\right\rangle\right\rangle\left\langle\beta_{b}\right\rangle$. We interpret Figs. 9(a) and 9(c) as follows: when $\Theta<0$, corresponding to good market sentiment and possible rate increase, the dynamics before the announcement have small $\beta_{b}$ and small $\Omega_{b}$ reflecting low activity. After the announcement, the values of $\beta_{a}$ and $\Omega_{a}$ increase, corresponding to a fast response of medium size. In the case of $\Theta>0$, corresponding to bad market sentiment resulting from speculation of a rate cut, the dynamics before the announcement have large $\beta_{b}$ and large $\Omega_{b}$, corresponding to a strong but quick buildup of volatility. After the announcement, the dynamics have large $\beta_{a}$ and small $\Omega_{a}$, corresponding to a strong and lasting relaxation dynamics. The interpretation of Figs. 9(b) and 9(d) is similar to the interpretation of Figs. 9 (a) and $9(\mathrm{c})$, in that both surprise $(\Delta>0)$ and expected $(\Delta$ $\approx 0$ ) bad news correspond to a stronger and longer-lasting relaxation dynamics. 


\section{S\&P 100}
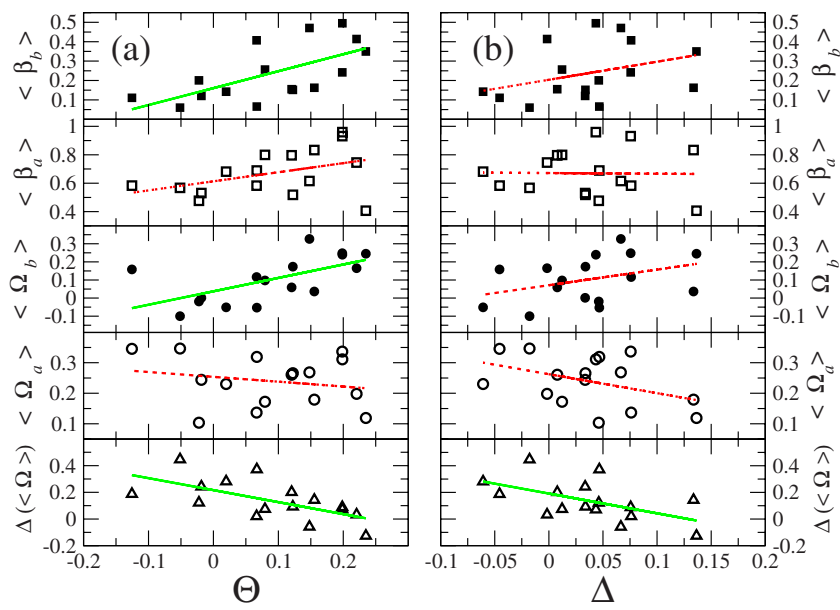

Bank sector
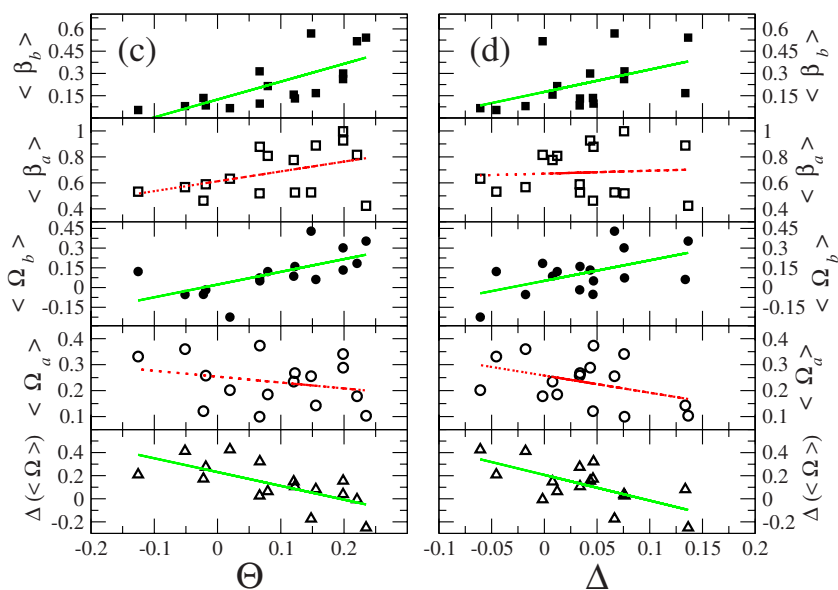

FIG. 9. (Color online) The relation between the size of the financial shock, quantified by the S\&P 100 volatility Omori-law parameters $\left\langle\beta_{b}\right\rangle,\left\langle\beta_{a}\right\rangle,\left\langle\Omega_{b}\right\rangle,\left\langle\Omega_{a}\right\rangle$, and $\Delta(\langle\Omega\rangle)=\left\langle\Omega_{a}\right\rangle-\left\langle\Omega_{b}\right\rangle$, and the size of the FOMC news, quantified through the metrics $\Theta$ representing market anticipation and $\Delta$ representing market surprise. All trends are consistent with the hypothesis that a strong anticipation of an interest-rate change, and the element of surprise inherent in the FOMC decision, results in a market perturbation that is significant in scale, and broad across the market. Linear regressions of (a) and (b) S\&P 100 data, and (c) and (d) bank sector data are provided for visual aid. Linear regressions that pass the ANOVA $F$ test (rejecting null hypothesis that regression slope $m=0$ ) at the $\alpha=0.05$ significance level are solid green line; regressions that fail to pass the $F$ test at the $\alpha=0.05$ significance level are dashed red line.

\section{DISCUSSION}

Information flows through various technological avenues, keeping the ever-changing world up to date. All news carries some degree of surprise, where the perceived magnitude of the news certainly depends on the recipient. In financial markets, where speculation on investment returns results annually in billions of dollars in transactions, news plays a significant role in perturbing the complex financial system both on large and small scales, reminiscent of critical behavior with divergent correlation lengths [47]. Perturbations to the financial system are easily transmitted throughout the market by the long-range interactions that are found in the networks of market correlations [31-33]. Afterward, the effects of the perturbation may persist via the long-term memory observed in volatility time series [39-41], with fluctuation scaling obeying the empirical Taylor's law $[48,49]$.

We have shown that the Omori law describes the dissipation of information following the arrival of Federal Open Market Commission (FOMC) news. This type of relaxation is consistent with the substructure of financial crash aftershocks observed on various scales [10]. In particular, we systematically study the dynamical response of the stock market to perturbative information in the form of a Federal Reserve FOMC interest-rate announcements, which can be expected (scheduled) or unexpected (as in cases of emergency).

In the case of unexpected news, as in Fig. 4(d), a pronounced response may result from reduced market liquidity since traders do not have ample time to prepare and adjust [12]. Our findings suggest that the dynamics of "rallies" based on other forms of news, such as earning reports, upgrades and downgrades of stocks by major financial firms, unemployment reports, merging announcements, etc., might also be governed by the Omori law with parameters that depend on the type of news. The impact of macroeconomic news has been analyzed for foreign exchange markets [12], where it is found that high levels of volatility are present following both scheduled and surprise news.

According to the efficient market hypothesis [14], the time scale over which news is incorporated into prices should be very small. However, consistent with previous studies, we find market under-reaction [13] evident in the finite time scale (found here to be at least one trading day) over which the volatility aftershocks are significant. Moreover, we quantify the dynamics before and after and show that the Omori parameters are related to investor sentiment [13], measured here by comparing the 6 month Treasury Bill and the Federal Funds rates.

It is also conceivable that Omori-law decay of market aftershocks also exists in the traded volume time series and the bid-ask spread time series [42,50]. Recently, Joulin et al. [36] used a similar method to describe the relaxation of trading following news streaming from feeds such as Dow Jones and Reuters and compared to the relaxation following anomalous volatility jumps. Joulin et al. [36] also found Omori-law relaxation, with exponent $\Omega_{a} \approx 1$ following a news source and $\Omega_{a} \approx 0.5$ following an endogenous jump; interestingly, they found that the amplitude of the Omori law is larger for news sources than for endogenous jumps. For further comparison, Weber et al. [10] found $\Omega_{a} \approx 0.69$ for the 38 days following the market crash on September 11, 1986. One distinct difference between these studies is the source of the news: Joulin et al. pooled together thousands of news sources, some possibly pertaining to only a single stock; we focus on one particular type of news, the FOMC target rate decision, which has a broad impact on the whole market and economy. It is possible that the difference between anticipated news and idiosyncratic news is the important criterion to consider when analyzing market response functions in re- 
lation to exogenous events. Here, we find unique dynamics before anticipated announcements.

In the case of FOMC news, speculation can be quantified by measuring the relative difference between the effective Federal Funds rate and the Treasury Bill in the weeks leading up to a scheduled meeting. We develop a speculation metric $\Theta$ and relate it to $V$, the volatility on the day of the meetings, finding that the market behaves more erratically when the Treasury Bill predicts a decrease in the Federal Funds target rate. A rate decrease often occurs in response to economic shocks, whereas a rate increase is often used to fight inflation. Hence, the asymmetric response in Fig. 3 to rising and falling rates is consistent with the sign effect, where it has been found that bad news causes a larger market reaction than good news [12], and that the asymmetry may result from the increased uncertainty in expectations among traders.

We analyze the four Omori-law parameters $\Omega_{b}, \Omega_{a}, \beta_{b}$, and $\beta_{a}$ calculated for 19 FOMC meetings. We conjecture that the Omori-law parameters are related to the market's speculation, anticipation, and surprise on the day of the FOMC meeting. In order to quantify speculation of rate cuts and rate increases, we define the measure $\Theta$, which is the relative spread between the Treasury Bill and the Federal Funds rates, before the meeting. In order to quantify surprise, we develop $\Delta$, which measures the change in the relative spread between the Treasury Bill and the Federal Funds rates, before and after the meeting. We relate both $\Theta$ and $\Delta$ to the dynamical response of the market on the day of the meeting. We find that relatively small $\Omega$ values and relatively large amplitude $\beta$ values, corresponding to longer relaxation time and large response, follow from bad news, as in the case of the market reaction to the World Trade Center attacks in 2001. All in all, these results show that markets relax according to the Omori law following large crashes and Federal interest-rate changes, suggesting that the perturbative response of markets belongs to a universal class of Omori laws, independent of the magnitude of news.

\section{ACKNOWLEDGMENTS}

We thank L. DeArcangelis and M. Levy for helpful suggestions and NSF for financial support.

567 (2009)

[20] J. B. Calrson, B. R. Craig, and W. R. Melick, J. Futures Markets 25, 1203 (2005).

[21] M. Piazzesi and E. Swanson, J. Futures Markets 55 (4), 677 (2008).

[22] K. N. Kuttner, J. of Monetary Economics 47, 523 (2001).

[23] S. Kwan, FRBSF Economic Letter 15, 1 (2007).

[24] J. B. Carlson, B. Craig, P. Higgins, and W. R. Melick, Economic Commentary of the Federal Reserve Bank of Cleveland (Fed. Reserve Bank, Cleveland, 2006), pp. 1-3.

[25] For the calculation of $\Theta$ and $\Delta$, we choose values of $L$ and $\lambda$ to be on the order of a couple of trading weeks prior to the announcement, so that we isolate fresh speculation leading into the meeting. The parameter $\lambda$ provides an effective cutoff period, after which the weights begin to decrease quickly. Conversely, the weights corresponding to days close to the meeting, $\Delta t=0$, are effectively constant. The values of $\Theta$ and $\Delta$ do not change much with varying choice of $L \in[5,15]$ or $\lambda$ $\in[5,15]$. Without loss of generality, we choose $L=15$ days and $\lambda=10$ days.

[26] B. Podobnik, D. Horvatic, A. M. Petersen, and H. E. Stanley, Proc. Natl. Acad. Sci. U.S.A. 106, 22079 (2009).

[27] We find historical information about FOMC meetings using resources at the Federal Reserve website and using newspaper archives. Intraday time of announcement, $T$, is often quoted in New York Times finance articles by Richard W. Stevenson the day after FOMC announcements. They are also evident in the intraday Omori plots of $N(t)$ in Fig. 4.

[28] K. Yamasaki, L. Muchnik, S. Havlin, A. Bunde, and H. E. Stanley, Proc. Natl. Acad. Sci. U.S.A. 102, 9424 (2005).

[29] F. Wang, K. Yamasaki, S. Havlin, and H. E. Stanley, Phys. Rev. E 73, 026117 (2006).

[30] F. Wang, P. Weber, K. Yamasaki, S. Havlin, and H. E. Stanley,

[18] J. D. Hamilton and O. Jorda, J. Polit. Econ. 110, 1135 (2002). 
Eur. Phys. J. B 55, 123 (2007).

[31] R. N. Mantegna, Eur. Phys. J. B 11, 193 (1999).

[32] L. Laloux, P. Cizeau, J. P. Bouchaud, and M. Potters, Phys. Rev. Lett. 83, 1467 (1999).

[33] V. Plerou, P. Gopikrishnan, B. Rosenow, L. A. Nunes Amaral, and H. E. Stanley, Phys. Rev. Lett. 83, 1471 (1999); V. Plerou, P. Gopikrishnan, B. Rosenow, Luis A. Nunes Amaral, T. Guhr, and H. E. Stanley, Phys. Rev. E 65, 066126 (2002).

[34] D. Sornette and A. Helmstetter, Physica A 318, 577 (2003).

[35] D. Sornette, Y. Malevergne, and J. F. Muzy, Risk 16, 67 (2003).

[36] A. Joulin, A. Lefevre, D. Grunberg, and J. P. Bouchaud, Wilmott Magazine 46, 1 (2008); e-print arXiv:0803.1769.

[37] O. Guedj and J. Bouchaud, IJTAF 8 (7), 933 (2005)..

[38] R. Crane and D. Sornette, Proc. Natl. Acad. Sci. U.S.A. 105, 15649 (2008).

[39] R. Cont, M. Potters, and J. P. Bouchaud, in Scale Invariance and Beyond, edited by B. Dubrulle, F. Graner, and D. Sornette (Springer, Berlin, 1997).

[40] Y. Liu, P. Cizeau, M. Meyer, C.-K. Peng, and H. E. Stanley, Physica A 245, 437 (1997); Y. Liu, P. Gopikrishnan, P. Cizeau, M. Meyer, C.-K. Peng, and H. E. Stanley, Phys. Rev. E 60, 1390 (1999).
[41] P. Gopikrishnan, V. Plerou, L. A. Nunes Amaral, M. Meyer, and H. E. Stanley, Phys. Rev. E 60, 5305 (1999).

[42] A. G. Zawadowski, G. Andor, and J. Kertész, Quant. Finance 6, 283 (2006).

[43] In Fig. 4 we plot $N(t)$, where $t=0$ corresponds to the opening bell at 9:30 a.m. Quantitatively, we calculate the Omori exponents using the displaced time $\tau$ from the announcement time $T$. In terms of the displaced time $\tau \equiv\left|t-T_{i}\right|$, we separate $N_{i}(t)$ into two separate time series $N_{b, i}(\tau)=N_{i}\left(T_{i}\right)-N_{i}\left(\left|t-T_{i}\right|\right)$, where $p<t<T_{i}$ and $N_{a, i}(\tau)=N_{i}\left(t-T_{i}\right)-N_{i}\left(T_{i}\right)$ with $t>T_{i}$. The parameter $p$ is a "padding" that eliminates the last $60 \mathrm{~min}$ of $N_{b, i}(\tau)$ [the first $60 \mathrm{~min}$ of $N_{i}(t)$ ], which eliminates opening effects and improves the regression analysis around $T_{i}$.

[44] D. Morse, J. Account. Res. 19, 374 (1981).

[45] O. Kim and R. E. Verrecchia, J. Account. Res. 29, 302 (1991).

[46] A. M. Petersen, F. Wang, S. Havlin, and H. E. Stanley, e-print arXiv:1006.1882.

[47] H. E. Stanley, Rev. Mod. Phys. 71, S358 (1999).

[48] Z. Eisler and J. Kertész, Phys. Rev. E 73, 046109 (2006).

[49] Z. Eisler, I. Bartos, and J. Kertész, Adv. Phys. 57, 89 (2008).

[50] A. Ponzi, F. Lillo, and R. N. Mantegna, Phys. Rev. E 80, 016112 (2009). 